\title{
Notes for Clinical Use of Extra Acupoints
}

\author{
Tong-zheng Hong* \\ As-You-Wish Healthcare Institute, Taiwan
}

Submission: August 23, 2018; Published: September 26, 2018

*Corresponding author: Tong-zheng Hong, As-You-Wish Healthcare Institute, Taiwan, Tel: 093-3086-399; Email: ty8876@ms24.hinet.net

\section{Abstract}

Pattern identification is the key concept of the traditional Chinese Medicine theories, which unfortunately cannot apply to extra acupoints for the lack of Yin-Yang. The recent studies suggest that extra acupoints selected in combination with traditional acupoints on the traditional fourteen meridians for anesthesia in surgical operation are marking the beginning of a new chapter and indicate more and more extra acupoints will be discovered.

Keywords: Yin-Yang; Extra acupoint; Pattern identification; Traditional chinese medicine

\section{Introduction}

Extra acupoints distinguish themselves from the regular acupoints on the traditional fourteen meridians with the unique indications, actions, and the great effectiveness in acupuncture theory and treatment, even though some of extra acupoints have not been verified with scientific evidence [1].

The Yin-Yang balance is the unique concept and the dominating key of the traditional Chinese medicine (TCM) throughout the history of Chinese medicine for many centuries [1]. It is believed that this concept absolutely distinguishes TCM from the Western medicine. Based on this concept, a disease thus indicates the loss of the balance of Yin-Yang. On the other hand, this concept also serves as the foundation and the guidelines for the etiology of diseases, diagnosis, and treatments [2].

The balance of Yin and Yang can be achieved by stimulations through techniques such as acupuncture, acupressure, moxibustion, cupping, and Tui Na on acupoints. The classic and well-known protocol of Four Gates (LV 3, Yin and LI 4, Yang) used for the successful treatment of the sub-health demonstrates the application of the concept and highlights the importance of YinYang balance [3].

However, extra acupoints are totally excluded from this theory simply because they have not been incorporated into the traditional fourteen meridians.

\section{Overview of the Development of Extra Acupoints}

The meridian system is established on the experience and knowledge of the use of extra acupoints and Ashi points. Ashi (Ah Shi) points, also called painful points, were first discovered accidently in ancient China to alleviate pain and treat certain sicknesses in the daily life. Literally in Chinese, Ashi meaning "Ah yes" is coined to refer to the certain "tender spot or pathological sites" on the body with responding signal of pain for diagnosis first presented in Jing Jin Di Shi San (Thirteenth Writing: Sinew Channels) of Ling Shu. In addition, Ashi points can also serve as a treatment site as well [4].

The entire Chinese medicine systems was not established and presented until the Warring States Period (476-221 BC). Examples of extra acupoints like "tip of finger poking", "between eyebrows", and "stabbing the Shaoyin beneath the tongue" were mentioned in Huang Di Nei Jing (Yellow Emperor's Inner Classic) with the locations of extra points, instead of the formal names [4].

Ashi points were not formally recognized and accepted to be incorporated into the traditional fourteen meridians. However, Extra acupoints themselves derived from Ashi points and represent the frequently used Ashi points with verified functions finally [1].

The specific names of extra points viewed as Ashi points initially were not given until the Sui Dynasty (581-618 CE) and the Tang Dynasty (618-907 CE). Up to 187 extra points were incorporated into Qian Jin Yao Fang (Thousand Ducat Formulas) by Sun Si-miao (581-682 CE) in the Tang Dynasty. Not until the Ming Dynasty were extra points formally discussed in the classic Qi Xiao Liang Fang (Wonderful Well-Tried Recipes). There were 26 extra points collected in the Chapter Extra Point in Volume 5, which marked the beginning of collecting extra points. 35 extra points were collected in Zhen Jiu Da Cheng The Systematic Classic of Acupuncture and Moxibustion); 84 extra points collected in Lei Jing Tu Yi (Illustrated Supplement to the Classic of Categories); 144 extra points collected in Zheng Jiu Ji Cheng (Compilation of Acupuncture of Moxibustion). Extra acupoints were officially separated from the traditional 14 meridians when the TCM doctors of the Tai Yi Yuan (Imperial Medical Institute) in the Qing 
Dynasty (1644-1911 CE) revised Yi Zong Jin Jian (Golden Mirror of Medicine) and built its own unique system [5].

Literature shows that most of the extra acupoints are not associated with a specific meridian and bear the characteristic of Yin-Yang; however, some extra acupoints like Yintang (M-HN-3), Sishencong (, M-HN-1) are located on the Governing vessel, one of the Erbai (M-UE-29) is on the PC meridian, and Taiyang (M-HN-9) is on the Sanjiao meridian [1].

\section{Keys to Therapeutic Effects}

Since diseases are understood to be a loss of balance between Yin and Yang as shown in Figure 1, good results cannot be expected without the positive consideration of Yin and Yang [1,2].

\begin{tabular}{|cc|}
\hline Yin & Yang \\
\hline & \\
Blood & Qi \\
Material & Function \\
Zang & Fu \\
Yin meridian & Yang meridian \\
\hline
\end{tabular}

An extra acupoint may be or may not on the meridians closely associated with Yin and Yang. The typical examples to explain the importance and necessity of Yin-Yang are Five Shu-Points. As a matter of fact, each Five Shu-Point corresponds to one of the specific phases of the Five Elements, highlighting the importance of balanced Yin -Yang.

The Five Elements theory and the Yin-Yang concept unfortunately cannot apply to extra aupoints only because they are not on the traditional fourteen meridians. In other words, extra acupoints like Yintang (M-HN-3), Sishencong (M-HN-1), Erbai (MUE-29), and Taiyang (M-HN-9) may be easier for practitioners to consider in practice with the location associated with the specific meridians bearing the characteristic of Yin-Yang as shown in Figure 1 [1].

Signs and symptoms in Traditional Chinese medicine may be totally different from those in the Western medicine and are understood to be broader. In general, TCM physicians or acupuncture practitioners usually do not follow the typical
Western pathological classifications of diseases, but rather rely on the patterns individualized by the imbalance of Yin-Yang, Qi, and Blood, and body fluids in the body [1].

Patterns are unique in TCM and pattern identification plays the most critical factor for the treatment and correlates closely with the successful outcomes. However, studies by Hong [1], Zhu et al. [6], Yu SY, et al. [7], and Paraskeva, et al. [8] show extra acupoints can be used together with traditional acupoints in clinical practice but patterns were unfortunately not included in the research designs.

One of the most practical approaches in a clinical practice to acupuncture involves the use of Ashi points when the patients suffer acute pain. However, this apparent simplicity may be discarded by practitioners when it comes to the application of TCM theories to treatment based on the complex meridians and the acupoint theory.

The historical developments of extra acupoints show an extra acupoint can surely play an essential role and be used alone for the treatment, even though they do not have the corresponding phases based on the Five Elements theory. Whether or not extra points can be selected in combination with traditional acupoints in a protocol for the more effective results of the treatments deserves attention.

\section{Discussion}

The challenge acupuncture practitioners face is when it is the best time to consider extra acupoints in a protocol.

The protocol following the TCM theories can bring about the best results. Taiyang (M-HN-9), for example, can be selected for one-sided headache as GB20 when the pathogen is exterior Wind [1]. In other words, this example explains the concept of pattern (Zeng) identification based on differential diagnosis is out of doubt the key to treatment using extra acupoints.

The historical developments of extra points show that an extra point can surely play an essential role in acupuncture and be used alone or with the traditional regular acupoints for the treatment. Unfortunately, the actions and indications of extra acupoints have not be scientifically researched and verified as traditional regular acupoints.

\begin{tabular}{|c|c|}
\hline Ta only & Ea only \\
\hline Ea $+\mathrm{Ta}$ & Ea $+\mathrm{Ta}$ \\
BUT & BUT \\
No. of Ea $>$ No. of $\mathrm{Ta}$ & No. of $\mathrm{a}>\mathrm{No}$. of $\mathrm{Ta}$ \\
\hline
\end{tabular}

Figure 2: Protocol using traditional acupoints and extra acupoints. Note: Ea for Extra acupoints and Ta for Traditional acupoints.

In strategy, a practitioner may take the principle of "Least needling, best results" into consideration in practice, keeping patients from pain or worry. The goal can be achieved with the following four protocols shown in Figure 2 when it comes to the tactic. 
As one of the oldest forms of the natural healing arts, acupuncture began its development and employment for anesthesia in surgical operation in the late1950's. In a pilot study in 2015 by Wang et al., 12 parents were randomized to an acupressure bead with occlusive tape covering at the Extra 3 (Yintang) point for 20 minutes. Parents in the acupressure group had significantly less anxiety at 20 minutes post-intervention compared with parents in the sham group [9].

It is true that patients and healthcare providers are usually concerned about side effects. The finding of a study on the Severity of Venipuncture Pain Among Hospitalized 6-12-Year-Old Children shows extra point Extra 3 (Yintang) in combination with P-8 (Laogong) point are recommended for its greater safety, costeffectiveness, and applicability [10].

The positive result of facial acupuncture reported by Donoyama et al. showed the protocol of BL1, GB1, ST1, ST3, ST4, ST7, SI19, CV24, Ex-HN3 (Yintang) and Ex-HN4 (Yuyao) could increase the water and oil content of the facial skin [11].

In clinic, practitioners are also concerned about if there is enough evidence-based research at present to show whether or not the use of extra acupoints alone will bring better results than the use in combination with regular acupoints. However, current studies show the extra acupoints can be effective alone, but better results of extra acupints used in combination with traditional acupoints are also reported in some researches [1].

There is no doubt that needling sensation (deqi) has been considered by many acupuncture practitioners to be a key component of a successful acupuncture treatment. However, this sensation will not occur when the needles are not insert into the acupoints accurately, which may also happen to the use of extra acupoints. In other words, locating an extra acupoint accurately is the key to the successful treatments. It is same with the development of the regular acupoints, location description may vary in different textbooks. For this issue, it is highly suggested that the use of a Moxa roll can help practitioners locate the extra acupoints accurately. The heat emitted from the Moxa roll penetrate the extra acupoint like an acupuncture needle as long as the Moxa roll pinpoints the acupoint location.

The well-known school of acupuncture characterized with extra acupoints in the world is Tung's acupuncture, which develops and establishes the exclusive theory and protocols extremely different from the TCM. In the meantime, the new extra acupoint Gangshui discovered and used for treating subacute cough demonstrates numerous extra acupoints may remain undiscovered [1].

Looking back at the development of extra acupoints, we can expect that more and more extra acupoints in various contexts may be discovered in the future.

Both acupuncture and acupressure are used based on the acupoint stimulation to achieve the desired results of treatments.
Acupuncture performed by acupuncturists to triggers a stronger stimulation on the acupoints than acupressure to activate the body's innate healing ability. Acupressure refers to stimulating the points on the surface of the skin by pressing with the hands, fingers, elbows or feet, is basically noninvasive and can be performed by the patients themselves.

Massage on extra acupoints is feasible for healthcare. Compared with acupuncture that may cause side effects suggested by Kashefi [12], acupressure has several advantages over acupuncture and deserves recommendations for its immediate effect, safety, costfree, no side effects, and convenience to patients.

\section{Conclusion}

With new extra acupoints increasingly discovered, it is predictable that acupuncture will gain more popularity all over the world in the future because it is effective, low cost, non-surgical, and painless way to healthcare.

On the other hand, additional researches are needed to understand if extra points can outperform the traditional acupoints on the traditional fourteen meridians.

\section{References}

1. Hong TZ (2017) Exploring a New Extra Point for Sub-acute Cough: A Case Report. Scholar's Press, Germany.

2. Maciocia G (1989) The foundations of Chinese Medicine. Library of Congress Cataloging in Publication Data, NY, USA.

3. Hong TZ (2009) Application of Four Gates to Treating Sub-health. Hsinchu City Community Policing 15: 13-17.

4. Shen-nong (2002) Development of Acupuncture and Moxibustion in China.

5. Kuo S J, Wang N (2018) Systematic Review of Extra Points.

6. Zhu ML, Jiang HC, Zeng HW (2016) Acupuncture combined with acupoint injection for 25 cases of post-cold cough. World Journal of Acupuncture - Moxibustion 26(4): 65-67.

7. Yu SY, Yang J, Yang MX, Yan G, Chen J, et al. (2015) Application of Acupoints and Meridians for the Treatment of Primary Dysmenorrhea: A Data Mining-Based Literature Study. Evidence- Based Complement and Alternative Medicine.

8. Paraskeva A, Melemeni A, Petropoulos G, Siafaka I, Fassoulaki A (2004) Needling of the extra 1 point decreases bis values and preoperative anxiety. Am J Chin Med 32(5): 789-794.

9. Wang SM, Gaal D, Maranets I, Caldwell-Andrews A, Kain ZN (2005) Acupressure and preoperative parental anxiety: a pilot study. Anesth Analg 101(3): 666-669.

10. Pour PS, A GF, KM JY (2017) Comparison of Effects of Local Anesthesia and Two-Point Acupressure on the Severity of Venipuncture Pain Among Hospitalized 6-12-Year-Old Children. J Acupunct Meridian Stud 10(3): 187-192.

11. Donoyama N, Kojima A, Suoh S, Ohkoshi N (2012) Cosmetic acupuncture to enhance facial skin appearance: a preliminary study. Acupunct Med 30(2):152-153.

12. Kashefi F, Khajehei M, Ashraf A, Jafari P (2010) Effect of acupressure at the Sanyinjiao point on primary dysmenorrhea: a randomized controlled trial. Complement Ther Clin Pract 16(4):198-202. 


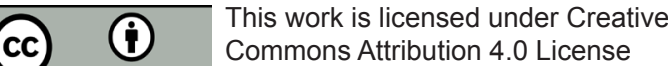

DOI: 10.19080/JCMAH.2018.08.555728

\section{Your next submission with Juniper Publishers} will reach you the below assets

- Quality Editorial service

- Swift Peer Review

- Reprints availability

- E-prints Service

- Manuscript Podcast for convenient understanding

- Global attainment for your research

- Manuscript accessibility in different formats

( Pdf, E-pub, Full Text, Audio)

- Unceasing customer service

Track the below URL for one-step submission https://juniperpublishers.com/online-submission.php 